\title{
Coupling-induced hysteresis in free-running oscillators
}

\author{
Almudena Suárez ${ }^{1}$, Robert Melville ${ }^{2}$, Franco Ramírez ${ }^{1}$ \\ ${ }^{1}$ University of Cantabria, Santander, Spain \\ ${ }^{2}$ EMECON LLC, New Providence, NJ, USA
}

\begin{abstract}
An investigation of coupling-induced hysteresis in free-running oscillators is presented, for possible application in sensors and RF identification (RFID). In its basic form, the phenomenon arises when an inductive element in an oscillator circuit gets magnetically coupled to another inductor in an external (passive) resonator. For a low mutual inductance or coupling, a small dip is observed in the oscillator amplitude curve versus a turning parameter. As demonstrated in this work, when the mutual inductance increases, the dip evolves into a hysteresis cycle. When coupled to several external resonators, the solution curve will also exhibit several hysteresis cycles about the corresponding resonance frequencies. Due to the complexity of the solution curves, a dedicated analysis methodology has been developed and tested on a prototype at $600 \mathrm{MHz}$, obtaining very good agreement between simulation and measurements.
\end{abstract}

Keywords — oscillator, hysteresis, stability.

\section{INTRODUCTION}

As has been known for almost one century, an oscillator circuit exhibits an amplitude change when approaching a resonant circuit tuned to its free-running frequency [1]-[2]. The phenomenon arises when an inductive element in the oscillator circuit gets magnetically coupled to the inductor in the external resonator. This property has been used in so-called "dip" oscillators to measure the resonant frequency of passive resonators or antennas [1]-[2]. The amplitude curve of the oscillator versus a tuning parameter exhibits a dip at the frequency of the external resonator. Despite being empirically known, there have been few works presenting a detailed investigation of the mechanism causing the amplitude dip.

In this work, the effects of coupling an oscillator circuit to an external resonator are theoretically analyzed. As will be analytically shown, when the mutual inductance increases, a hysteresis phenomenon [3] is induced in the oscillator amplitude and frequency versus the tuning parameter. The same effect can be achieved when coupling to distributed resonators. We believe that the capability to induce hysteresis in the response of an oscillator circuit by coupling it to one or more resonant networks can be of interest for passive RFID application [4], due to the enhanced sensitivity to the tag resonances associated with this phenomenon. In fact, the coupling to several resonators will give rise to several distinct hysteresis cycles, about their corresponding resonance frequencies. Additionally, work is in progress on an identification method in which jumps are induced to either one or another of the coexisting stable solutions of a single hysteresis loop, according to an encoded bit sequence of the tag.

Due to the difficulty involved in the analysis of multivalued curves with multiple hysteresis cycles, a novel methodology is proposed. This is based on an independent frequency-domain simulation of the oscillator core and the coupled passive network, both modeled in terms of admittance functions. Then, the two models are combined to compose the complete oscillator equation, solved through a contour-intersection procedure [5]. The analysis and synthesis method has been successfully applied to a BJT oscillator at $600 \mathrm{MHz}$.

\section{COUPLING EFFECTS}

In a first analytical investigation, a simple cubicnonlinearity oscillator (Fig. 1), with the passive elements $R, L$, $C$, is considered. The inductor $L$ is coupled to the inductor $L_{2}$ of an external parallel resonator (with loss) modeled by by $G_{2}, L_{2}$ and $C_{2}$. The equation system governing the coupled-circuit behavior is:

$$
\begin{aligned}
& I(V)+(G+j C \omega) V+I_{1}=0 \\
& V=j L \omega I_{1}+j M \omega I_{2} \\
& \left(G_{2}+j C_{2} \omega\right) V_{x}+I_{2}=0 \\
& V_{x}=j L_{2} \omega I_{2}+j M \omega I_{1}
\end{aligned}
$$

where $M$ is the mutual inductance, related to the inductor coupling factor $k$ and the inductor values as $M=k \sqrt{L L_{2}}$.

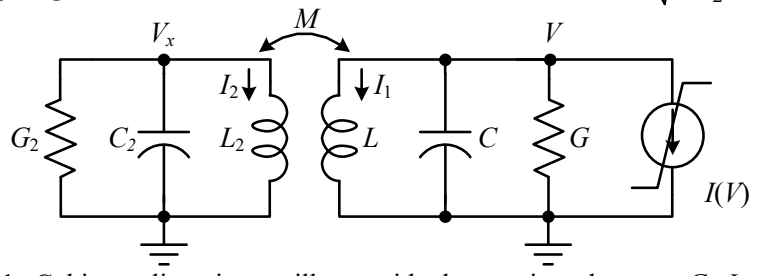

Fig. 1. Cubic-nonlinearity oscillator with the passive elements $G, L, C$, is considered. The inductor $L$ is coupled to the inductor $L_{2}$ of an external parallel resonator, composed by $G_{2}, L_{2}$ and $C_{2}$.

Equations (a) and (b) correspond to the oscillator circuit and (c) and (d) to the coupled resonator. Solving for $I_{2}$ in terms of $I_{1}$ from (c) and (d), and for $I_{1}$ from (b), and replacing in (a), one obtains:

$$
G_{N}(V)+(G+j C \omega)+\frac{1}{j L \omega+\frac{M^{2} \omega^{2}\left(G_{2}+j C_{2} \omega\right)}{1+j L_{2} \omega\left(G_{2}+j C_{2} \omega\right)}}=0
$$

where $G_{N}(V)=I(V) / V$. One easily gathers that the coupling gives rise to a change of the equivalent load admittance, which changes from $1 /(j L \omega)$ to the third term in (2). Splitting (2) into real and imaginary parts, one obtains the following system:

$$
\begin{aligned}
H_{r}= & \left(1-k^{2}\right) L C L_{2} C_{2} \omega^{4}+ \\
& {\left[\left(k^{2}-1\right) L L_{2} G_{2} G_{T}(V)-L_{2} C_{2}-L C\right] \omega^{2}+1=0 } \\
H_{i}= & \left(k^{2}-1\right) L L_{2}\left[G_{T}(V) C_{2}+G_{2} C\right] \omega^{2}+ \\
& G_{T}(V) L+G_{2} L_{2}=0
\end{aligned}
$$


where $G_{T}(V)=G_{N}(V)+G$. Using (b), one can solve for $\omega^{2}$ in terms of $G_{T}(V)$. Then, using (a), one can solve for $G_{T}(V)$ in terms of the circuit elements. In particular, the function $G_{N}(V)=a+3 / 4 b V^{2}$, where $a<0$ and $b>0$, will be assumed. Tracing $\omega$ and $V$ versus $C$ under variations of the coupling factor $k$, one obtains the curve family in Fig. 2. Results obtained with commercial HB (whenever convergence is possible) are superimposed with excellent agreement. To understand the curve evolution, one should note that at the particular $C$ value that fulfills: $1 /(L C)=1 /\left(L_{2} C_{2}\right)=\omega_{2}^{2}$, the oscillation frequency of the original (isolated) oscillator agrees with that of the external resonator $\omega=\omega_{2}$. In these conditions, system (3) always exhibits a solution $\omega_{2}$, as can be seen in Fig. 2(a), where all the frequency curves intersect at $C=9.78 \mathrm{pF}, f=681 \mathrm{MHz}$. Using $1 /(L C)=1 /\left(L_{2} C_{2}\right)$ and $\omega=\omega_{2}$, and solving for the amplitude $V$, one obtains:

$$
V^{2}=\frac{4}{3 b}\left(-a-G-\frac{k^{2}}{\left(1-k^{2}\right)} \frac{1}{L L_{2} G_{2} \omega_{2}^{2}}\right)
$$

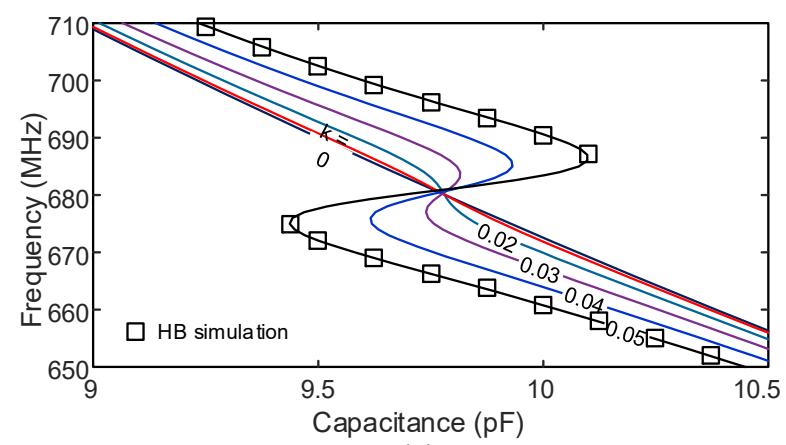

(a)

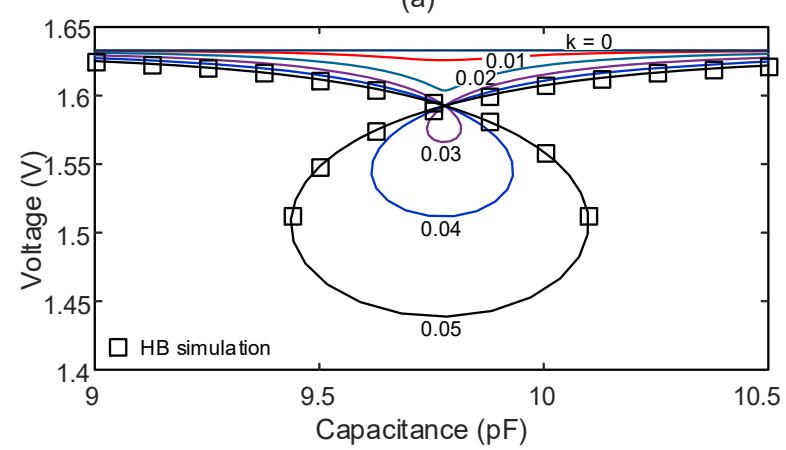

(b)

Fig. 2. Analytical investigation. Solution curves of system (3) traced versus $C$ under variations of the coupling factor $k$. Results obtained with commercial HB (whenever convergence is possible) are superimposed. (a) Oscillation frequency. (b) Oscillation amplitude.

Because the third term of (4) is negative (since $k<1$ ), there will be a reduction in the oscillation amplitude with respect to uncoupled conditions $(k=0)$, which explains the amplitude dip when $1 /(L C)=1 /\left(L_{2} C_{2}\right)$. In fact, there is a power transfer from the oscillator to the external resonator. The amplitude dip will be more pronounced for larger $k$ and smaller for larger $G_{2}$, in agreement with the curves of Fig. 2(b). However, from a certain $k$, system (3), having third order in $V$, can provide multivalued solution curves. Using $1 /(L C)=1 /\left(L_{2} C_{2}\right)$ in (3), one obtains three solutions: one at $\omega_{2}$ and two other solutions above and below this frequency, as shown in Fig. 2(a). The multivalued sections are bounded by turning points or infiniteslope points, at which the Jacobian matrix associated with system (3) becomes singular. Thus, the turning-point condition is the following:

$$
\operatorname{det}\left[\begin{array}{ll}
\frac{\partial H_{r}}{\partial V} & \frac{\partial H_{r}}{\partial \omega} \\
\frac{\partial H_{i}}{\partial V} & \frac{\partial H_{i}}{\partial \omega}
\end{array}\right]=0
$$

which should be fulfilled in combination with $H_{r}=0$ and $H_{i}=0$ in (3). Note that under some conditions, the multivalued curve can split into distinct (isolated) solution curves. A simple way to see this is to consider the case $G_{2}=0, L_{2}=L$ and $C_{2}=C$. Solving for $\omega$, one obtains two distinct oscillation frequencies:

$$
\omega_{1}=1 / \sqrt{C(L+M)} ; \quad \omega_{2}=1 / \sqrt{C(L-M)}
$$

The two oscillation frequencies converge to a single one when $M=0$. On the other hand, $\omega_{2}$ tends to infinite when $L=M$, which is impossible in practice, since $k<1$.

\section{ANALYSIS METHOD}

The analysis method is based on independent modeling of the coupled sub-circuit and the oscillator core, obtained at the terminals $T_{1}-T_{2}$, between which the inductor coupled to the external resonator is connected [Fig. 3(a)]. The method will be valid provided the waveform between $T_{1}$ and $T_{2}$ has a limited harmonic content, since it neglects the coupling effects at higher harmonic terms. To obtain the model of the oscillator core, an outer-tier admittance function $Y(V, \omega)$ is extracted by connecting an auxiliary generator (AG) $[6]$ between $\mathrm{T}_{1}$ and $\mathrm{T}_{2}$, as shown in Fig. 3(b). The high value parallel resistor $R_{\infty}$ is used to prevent any convergence problems. A double sweep is carried out in $V$ and $\omega$, performing a harmonic-balance (HB) simulation at each sweep step, with an many harmonic components as desired. As stated, the analysis error will come from the fact that coupling effects will only be considered at the fundamental frequency. Since the resonator action is dominant at this frequency, a small error can be expected. The model of the coupled subcircuit is calculated by obtaining its admittance function through a simple linear simulation, as shown in Fig. $3(\mathrm{c})$. The method is general and can be applied to any kind of coupled subcircuit, composed of one or more coupled resonators, which can be lumped or distributed. It is fully compatible with the use of electromagnetic simulators.

The method is illustrated with the simple case shown in Fig. 3(c), though, as stated, it can be generalized to lumped and coupled networks of arbitrary complexity. It corresponds to a lumped resonator $G_{2}, C_{2}, L_{2}$, where $L_{2}$ is coupled to the inductance $L$ of an arbitrary oscillator, modeled with $Y(V, \omega)$. Note that the oscillator inductor $L$ is excluded from $Y(V, \omega)$. The circuit equations are:

$$
\begin{array}{lll}
Y(V, \omega) V+I_{1}=0 & (\mathrm{a}), & \left(G_{2}+j C_{2} \omega\right) V_{x}+I_{2}=0 \\
V=j L \omega I_{1}+j M \omega I_{2} & \text { (b), } & V_{x}=j L_{2} \omega I_{2}+j M \omega I_{1}
\end{array}
$$


And proceeding in a manner similar to (1)-(2), one obtains:

$$
Y_{T}(V, k, \omega)=Y(V, \omega)+G_{c}(k, \omega)+j B_{c}(k, \omega)=0
$$

where $Y_{c}(k, \omega)=G_{c}(k, \omega)+j B_{c}(k, \omega)$ is the admittance term that replaces the original oscillator admittance $-j /(L \omega)$. The complex equation describing the oscillator coupled to the external resonator is:

$$
Y_{T}(V, k, \omega)=Y(V, \omega)+Y_{c}(\omega, k)=0
$$

The solution points are obtained applying the contour intersection technique in [4]. This is based on the calculation of the intersection points of the two contours $\operatorname{Re}\left(Y_{T}\right)=0$ and $\operatorname{Im}\left(Y_{T}\right)=0$, and simultaneously provides all the coexisting periodic solutions. When considering an analysis parameter $\eta$ (belonging to the oscillator circuit), for a particular $k$ value, a triple sweep must be performed in $\eta, V$ and $\omega$, This way one obtains a collection of functions $Y(V, \omega, \eta)$, from which, in turn, one obtains $Y_{T}(V, \omega, \eta)$. The solution curve versus $\eta$ is provided by the set of intersection points: $S(\eta)=\left\{V, \omega \mid \operatorname{Re}\left[Y_{T}(V, \omega, \eta)\right]=0 \cap \operatorname{Im}\left[Y_{T}(V, \omega, \eta)\right]=0\right\}$. This procedure is very convenient for the calculation of relatively complex solution curves, in combination with commercial HB simulations, since it avoids multiple manual parameter switches and their associated convergence problems [7]. Actually, to obtain a hysteresis cycle, three manual parameter changes must be performed. In addition, in the case of inductive coupling, HB has been found to exhibit convergence difficulties near the hysteresis regions.

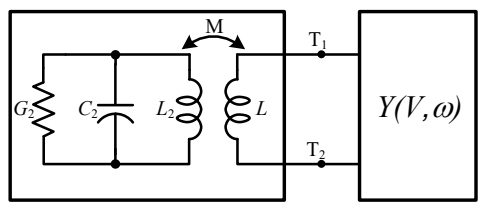

(a)

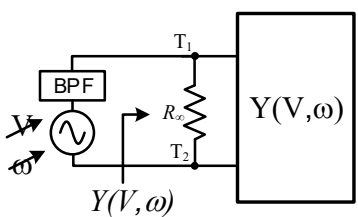

(b)

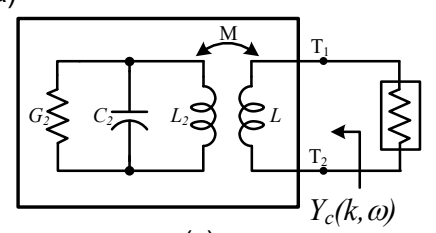

(c)
Fig. 3. Sketch of the analysis method, using the reference terminals $T_{1}-T_{2}$. The oscillator core is modeled in terms of the admittance function $Y(V, \omega)$. The coupled subcircuit is modeled with $Y_{c}(\omega)$. (a) Full circuit in the particular case of a lumped-element external resonator. (b) Extraction of $Y(V, \omega)$ using an AG. (b) Extraction $Y_{c}(\omega)$ through a linear analysis.

\section{COUPLING-INDUCED HYSTERESIS IN COLPITTS OSCILLATOR}

The analysis method has been applied to the Colpitts oscillator in Fig. 4. The schematic includes a transmission line that is coupled to an external resonator. In the absence of these coupling effects (and including the transmission line), the oscillation frequency is $f_{o}=600 \mathrm{MHz}$. The function $Y(V, \omega)$, defined between the terminals $T_{1}$ and $T_{2}$, shown in Fig. 4(a), is calculated replacing the coupled transmission line with a high resistor $R_{\infty}$. Fig. 5(a) presents the oscillation frequency variation versus the tuning voltage of the varactor diode $D_{1}$ when the transmission line is coupled to a distributed resonator having the resonant frequency $f_{r 1}=600 \mathrm{MHz}$. For the distance $s=2 \mathrm{~mm}$, one obtains a small hysteresis loop about the varactor bias voltage at which the oscillation frequency agrees with $f_{r 1}$. For the smaller distance $s=1 \mathrm{~mm}$ (stronger coupling), the hysteresis (about the same bias voltage) is more pronounced. In a second experiment, the oscillator transmission line is coupled to two external resonators, having the resonant frequencies $f_{r 1}=$ $540 \mathrm{MHz}$ and $f_{r 2}=640 \mathrm{MHz}$. As shown in Fig. 6, the coupling gives rise to two hysteresis cycles at the frequencies of the two resonators. Note that in this proof-of-concept, the distributed resonators are on the same substrate, but there is no conceptual difficulty in getting the same effect through air coupling, provided suitable antennas are used. At present, we are working in this direction.
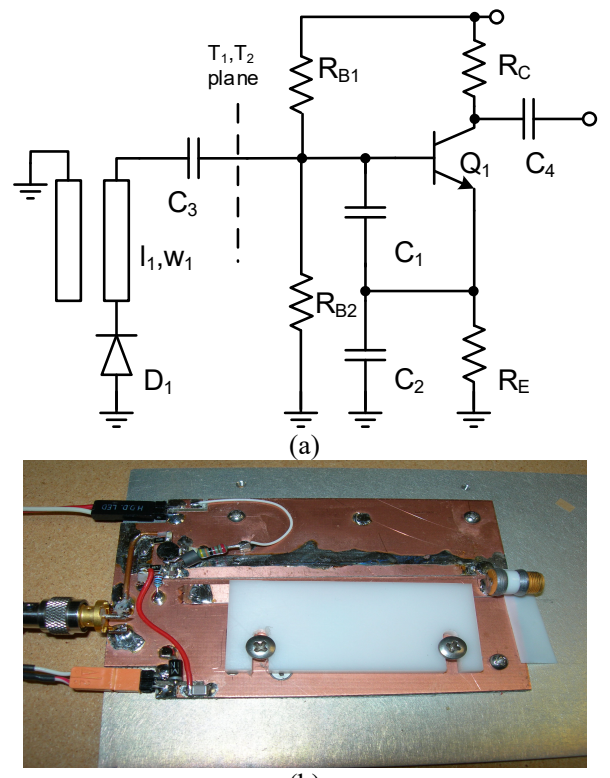

(b)

Fig. 4. Colpitts oscillator at $f_{o}=600 \mathrm{MHz}$, including a transmission line, coupled to one or two external distributed resonators. (a) Schematic, showing the plane $\mathrm{T}_{1}-\mathrm{T}_{2}$ used in Fig, 3. (b) Photograph.

The circuit of Fig. 4 was implemented as a prototype using ground-plane construction, using an MFR911 BJT. The resonator shown in the schematic was implemented with a strip of double-sided circuit board using a low-loss microwave substrate laminated onto the ground plane. See Fig. 4(b) for a photograph. Other components were realized with small surface-mount parts. This is an effective prototyping technique for frequencies up to L-band. Fig. 6 shows a hysteresis curve obtained by sweeping the tuning voltage across the varactor with another passive micro-strip resonator edge-coupled to the main micro-strip resonator of the oscillator. A plastic clamp was fabricated to hold the passive resonator at a fixed distance from the active resonator. As shown in [7], a varactor diode at large signal levels can exhibit self-modulation, which alters the effective capacitance, leading to a hysteresis effect. In order to demonstrate that such self-modulation was not the reason for the hysteresis of Fig. 6 we substituted a mechanical variable capacitor for the varactor and swept it over the same capacitance range. A qualitatively identical hysteresis curve 
was obtained demonstrating that the hysteresis is not attributable to self-modulation of the varactor diode. Alternatively, a different hysteresis curve was obtained by positioning a passive resonator implemented with lumped elements $4 \mathrm{~mm}$ above the varactor end of the active micro-strip resonator. Finally, Fig. 6 shows a hysteresis curve obtained by coupling two passive resonators to the active resonator of the Colpitts oscillator.

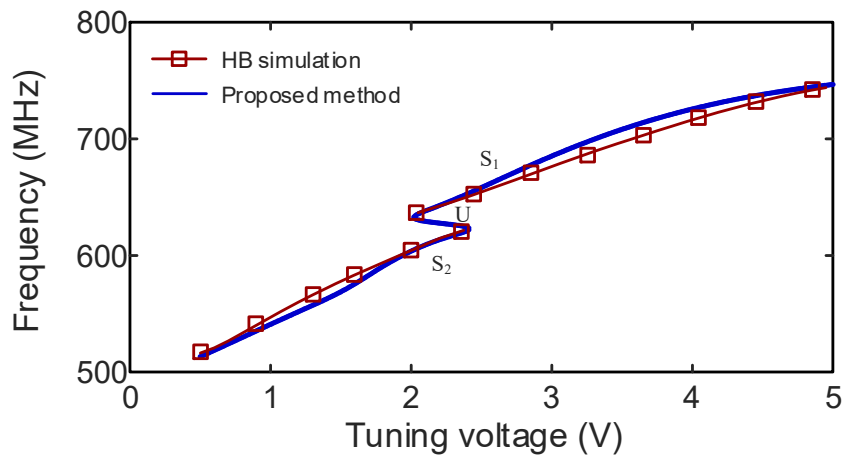

(a)

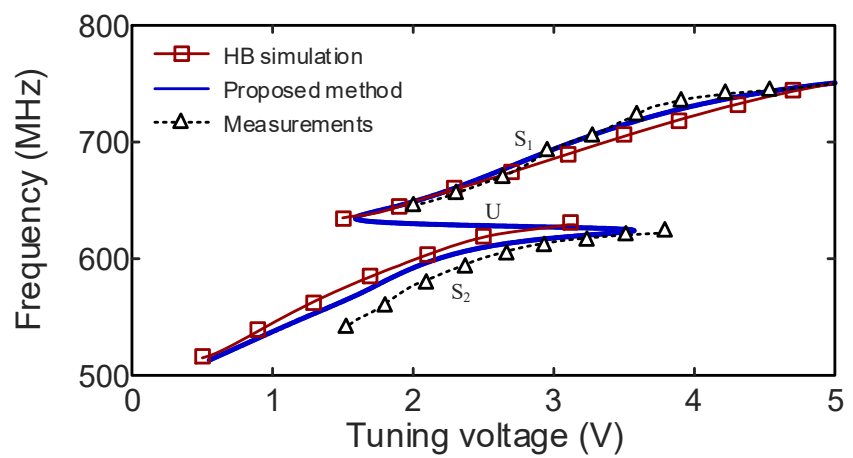

(b)

Fig. 5. Oscillator frequency curve versus the capacitor $C_{1}$, when coupled to the external distributed resonator. (a) For a coupling distance $s=2 \mathrm{~mm}$. Hysteresis. (b) For a coupling distance $s=1 \mathrm{~mm}$. Three distinct oscillation curves.

Although a detailed description is beyond the scope of this initial work, we have preliminary experimental results on a different encoding mechanism for chipless RFID tags [4], based on a single hysteresis loop, as in the case of Fig. 5(a). For tuning voltage in the middle (multvalued) section, there are three possible steady-states: $S_{1}, S_{2}$ and $U$. The states $S_{1}$ and $S_{2}$ are stable, whereas $\mathrm{U}$ is unstable. Extending the techniques of [8] to the autonomous case, it is possible to stabilize the oscillator in state $\mathrm{U}$. When the stabilization mechanism is turned off, the oscillator will jump (randomly) to one of the stable states $S_{1}$ or $\mathrm{S}_{2}$. Suppose, however, that an additional passive tag resonator tuned to the frequency of either $S_{1}$ or $S_{2}$ - is air-coupled to the oscillator, then the stabilization mechanism is turned off. In our experiments, we can make the oscillator then jump reliably to the corresponding stable steady state, even with relatively weak coupling of the tag resonator. Possibly, such a mechanism could be used to encode a binary bit. If the frequencies corresponding to $S_{1}$ and $S_{2}$ are well separated, we expect the reading process to be robust. Further experiments are in progress.

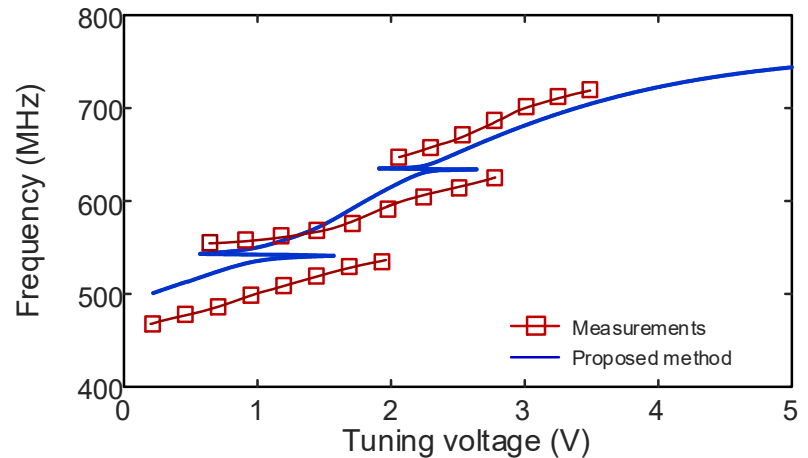

Fig. 6. Oscillator frequency curve versus the capacitor $C_{1}$, when coupled to two external distributed resonators.

\section{CONCLUSIONS}

An in-depth analysis of the effects of the inductive coupling of an oscillator to one or more external resonators has been presented. The study includes an analytical formulation for the understanding of these effects, which evolve from a dip in the amplitude curve traced versus a tuning parameter to a hysteresis phenomenon, when increasing the coupling strength. The coupling to several external resonators at distinct oscillation frequencies gives rise to a dip or hysteresis about the corresponding resonance frequencies. A novel analysis methodology has been proposed to avoid the difficulties associated with the simulation of solution curves containing several hysteresis cycles, which involve multiple manual parameter switches in commercial HB. This is based on an independent frequency-domain simulation of the oscillator core and the coupled passive network. The analysis and synthesis methods have been applied to a Colpitts oscillator coupled to one and two external resonators, obtaining very good qualitative agreement between simulation and measurements.

\section{ACKNOWLEDGMENT}

This work was supported by the Spanish Ministry of Science, Innovation and Universities under the research project TEC2017-88242-C3-1-R (ERDF/FEDER).

\section{REFERENCES}

[1] Elliott White, Laboratory Oscillators: A Short-wave Oscillator, American Radio League 1YB, May 1923.

[2] "The Amateur Radio Handbook", Third Ed., Radio Society of Great Britian, 28 Little Russel St, (1961), London, W.C.1, pp. 473-476.

[3] J. Guckenheimer, P. Holmes, Nonlinear Oscillations, Dynamical Systems and Bifurcations of Vector Fields. NY: Springer-Verlag, 1990.

[4] M. A. Islam, N. C. Karmakar, "Compact Printable Chipless RFID Systems," IEEE Trans. Microw. Theory Techn., vol. 63, no. 11, pp. 3785-3793, Nov. 2015.

[5] S. Hernández, M. Pontón, A. Suárez, "Simulation Method for Complex Multivalued Curves in Injection-Locked Oscillators," IEEE Trans. Microw. Theory Techn., vol. 65, no. 11, pp. 4046-4062, Nov. 2017.

[6] S. Sancho, F. Ramírez, A. Suárez, "General stabilization techniques for microwave oscillators", IEEE Microw. Wireless Compon. Lett., vol. 15, no. 12 , pp. $868-870$, Dec. 2005.

[7] L. Pantoli, A. Suárez, G. Leuzzi, F. Di Paolo, "Complete and Systematic Simulation Tools for Frequency Divider Design," IEEE Trans. Microw. Theory Techn., vol. 56, no. 11, pp. 2442-2452, Nov., 2008.

[8] R. C. Melville, A. Suárez, "Experimental Investigation of Bifurcation Behavior in Nonlinear Microwave Circuits," IEEE Trans. Microw. Theory Techn., vol. 65, no. 5, pp. 1545-1559, May 2017. 